PROCEEDINGS OF THE

AMERICAN MATHEMATICAL SOCIETY

Volume 130, Number 12, Pages 3609-3617

S 0002-9939(02)06680-7

Article electronically published on June 11, 2002

\title{
OPERATOR WEAK AMENABILITY OF THE FOURIER ALGEBRA
}

\author{
NICO SPRONK
}

(Communicated by David R. Larson)

Abstract. We show that for any locally compact group $G$, the Fourier algebra $\mathrm{A}(G)$ is operator weakly amenable.

Let $G$ be a locally compact group. It is shown by Johnson in [16] (and by Despić and Ghahramani in [6]) that the group algebra $\mathrm{L}^{1}(G)$ is always weakly amenable. It is natural to ask whether the same holds for the Fourier algebra $\mathrm{A}(G)$. In [17] it is shown for $G=\mathrm{SO}(3)$ that $\mathrm{A}(G)$ is not weakly amenable. We note that $\mathrm{A}(G)$ is weakly amenable (and, in fact amenable) whenever $G$ is Abelian, since $\mathrm{A}(G) \cong \mathrm{L}^{1}(\widehat{G})$ where $\widehat{G}$ is the dual group. Also, $\mathrm{A}(G)$ is weakly amenable whenever the connected component of the identity in $G$ is Abelian [11]. It is conjectured that this characterizes the weak amenability of $\mathrm{A}(G)$.

Since $\mathrm{A}(G)$ is the predual of the von Neumann algebra $\operatorname{VN}(G)$, it admits a natural structure as an operator space. Using this structure, Ruan [23] developed a completely bounded cohomology theory and proved that $\mathrm{A}(G)$ is operator amenable exactly when $G$ is an amenable group. This is analogous to Johnson's result [15] that $\mathrm{L}^{1}(G)$ is amenable exactly when $G$ is amenable. We note that the natural operator space structure on $\mathrm{L}^{1}(G)$ as the predual of $\mathrm{L}^{\infty}(G)$ is such that all bounded maps from $\mathrm{L}^{1}(G)$ into any operator space are automatically completely bounded. Thus the notions of amenability and operator amenability coincide on $\mathrm{L}^{1}(G)$, making Ruan's result truly a dual result of Johnson's, in the sense that $\mathrm{A}(G)$ is the dual of $\mathrm{L}^{1}(G)$ in a way which generalizes Pontryagin's Duality Theorem (see [9]).

The purpose of this note is to show that the natural operator space structure on $\mathrm{A}(G)$ allows us to obtain another analogous result to one for $\mathrm{L}^{1}(G)$ : we show that $\mathrm{A}(G)$ is always operator weakly amenable. We note that this result was obtained in [12], for the case that the connected component of the identity in $G$ is amenable.

The author would like to thank his doctoral advisor, Brian Forrest, for suggesting this problem.

\section{Preliminaries}

If $\mathcal{X}$ is a Banach space we always let $\mathcal{X}^{*}$ denote its dual and $\mathcal{B}(\mathcal{X})$ denote the Banach algebra of bounded operators on $\mathcal{X}$. The symbol $\mathcal{H}$ (possibly with a

Received by the editors July 6, 2001.

2000 Mathematics Subject Classification. Primary 46L07; Secondary 43A07.

Key words and phrases. Fourier algebra, operator space, weakly amenable Banach algebra.

This work was supported by an Ontario Graduate Scholarship. 
subscript) will always denote a Hilbert space and $\mathcal{U}(\mathcal{H})$ will denote the group of unitary operators on $\mathcal{H}$ with the relativized weak operator topology.

Let $G$ be a locally compact group. The Fourier and Fourier-Stieltjes algebras, $\mathrm{A}(G)$ and $\mathrm{B}(G)$, are defined in [10]. We recall that $\mathrm{B}(G)$ is the space of matrix coefficients of all continuous unitary representations of $G$; i.e. the space of functions of the form $s \mapsto\langle\pi(s) \xi \mid \eta\rangle$ where $\pi: G \rightarrow \mathcal{U}\left(\mathcal{H}_{\pi}\right)$ is a continuous homomorphism and $\xi, \eta \in \mathcal{H}_{\pi} . \mathrm{B}(G)$ is the dual of the enveloping group $\mathrm{C}^{*}$-algebra $\mathrm{C}^{*}(G)$ via $\langle a,\langle\pi(\cdot) \xi \mid \eta\rangle\rangle=\left\langle\pi_{*}(a) \xi \mid \eta\right\rangle$, where $\pi_{*}: \mathrm{C}^{*}(G) \rightarrow \mathcal{B}\left(\mathcal{H}_{\pi}\right)$ is the representation induced by $\pi$. It can be shown that $\mathrm{B}(G)$ is a commutative Banach algebra (under pointwise operations) and $\mathrm{A}(G)$ is the closed ideal in $\mathrm{B}(G)$ generated by compactly supported matrix coefficients.

If $\pi$ is a continuous unitary representation of $G$, let $\mathrm{A}_{\pi}$ be the norm closure of $\operatorname{span}\left\{\langle\pi(\cdot) \xi \mid \eta\rangle: \xi, \eta \in \mathcal{H}_{\pi}\right\}$ in $\mathrm{B}(G)$. Then, by [1, 2.2], $\mathrm{A}_{\pi}^{*} \cong \mathrm{VN}_{\pi}$, where $\mathrm{VN}_{\pi}$ is the von Neumann algebra generated by $\pi(G)$. If $\lambda$ is the left regular representation of $G$ on $\mathrm{L}^{2}(G)$, then $\mathrm{A}(G)=\mathrm{A}_{\lambda}$ and we write $\mathrm{VN}(G)=\mathrm{VN}_{\lambda}$. Given two unitary representations $\pi$ and $\sigma$ of $G$, we let $\pi \oplus \sigma: G \rightarrow \mathcal{U}\left(\mathcal{H}_{\pi} \oplus \mathcal{H}_{\sigma}\right)$ denote their direct sum. If $\pi$ and $\sigma$ are disjoint (i.e. there are no subrepresentations $\pi^{\prime}$ of $\pi$ and $\sigma^{\prime}$ of $\sigma$ such that $\pi^{\prime}$ is spatially equivalent to $\sigma^{\prime}$ ), then

$$
\mathrm{VN}_{\pi \oplus \sigma}=\mathrm{VN}_{\pi} \oplus_{\infty} \mathrm{VN}_{\sigma} \quad \text { and } \quad \mathrm{A}_{\pi \oplus \sigma}=\mathrm{A}_{\pi} \oplus_{1} \mathrm{~A}_{\sigma}
$$

by [22, 3.8.10] and [1,3.13], respectively, where $\oplus_{p}$ denotes the $\ell^{p}$-direct sum for $p=1, \infty$.

Our standard reference for operator spaces will be 8 . Given two operator spaces $\mathcal{X}$ and $\mathcal{Y}$, we denote by $\mathcal{C B}(\mathcal{X}, \mathcal{Y})$ the space of completely bounded linear maps between $\mathcal{X}$ and $\mathcal{Y}$ and denote the norm on it by $\|\cdot\|_{c b}$. If $\mathcal{X}=\mathcal{Y}$, we will denote the Banach algebra $\mathcal{C B}(\mathcal{X}, \mathcal{X})$ by $\mathcal{C B}(\mathcal{X})$. Dual spaces will always be given the standard operator dual structure ([8, Sec. 3.2], [3]). Given two operator spaces $\mathcal{X}$ and $\mathcal{Y}$, their direct product with the canonical product operator space structure will be denoted $\mathcal{X} \oplus_{\infty} \mathcal{Y}$. The direct sum $\mathcal{X} \oplus_{1} \mathcal{Y}$ will be given the operator space structure it obtains from being imbedded into $\left(\mathcal{X}^{*} \oplus_{\infty} \mathcal{Y}^{*}\right)^{*}$. The product and sum are denoted by $\mathcal{X} \oplus_{C M} \mathcal{Y}$ and $\mathcal{X} \oplus_{C L} \mathcal{Y}$, respectively, in [7. If $\mathcal{M}$ is a von Neumann algebra, its predual $\mathcal{M}_{*}$ will always be given the operator space structure it inherits from being imbedded in the dual $\mathcal{M}^{*}$. Moreover, $\mathcal{M}$ is then the standard dual of $\mathcal{M}_{*}($ [8, 4.2.2], 3] $)$. In particular, each space $\mathrm{A}_{\pi} \cong\left(\mathrm{VN}_{\pi}\right)_{*}$ will be endowed with this predual operator space structure.

The projective tensor product of Banach space theory admits an operator space analogue. Given two operator spaces $\mathcal{X}$ and $\mathcal{Y}$, we denote their operator space projective tensor product by $\mathcal{X} \widehat{\otimes} \mathcal{Y}$. We will not need the explicit formula for the norm of this tensor product, but note that it is a completion of the algebraic tensor product $\mathcal{X} \otimes \mathcal{Y}$. We will use two important properties of this operator tensor product. First,

$$
(\mathcal{X} \widehat{\otimes} \mathcal{Y})^{*} \cong \mathcal{C B}\left(\mathcal{X}, \mathcal{Y}^{*}\right) \quad \text { via } \quad\langle x \otimes y, T\rangle=\langle y, T x\rangle .
$$

See [8, 7.1.5] or [4. This is analogous to the usual dual formula for the Banach space projective tensor product. Second, if $\mathcal{M}$ and $\mathcal{N}$ are von Neumann algebras, then

$$
\mathcal{M}_{*} \widehat{\otimes} \mathcal{N}_{*} \cong(\mathcal{M} \bar{\otimes} \mathcal{N})_{*}
$$


where $\mathcal{M} \bar{\otimes} \mathcal{N}$ is the von Neumann tensor product of $\mathcal{M}$ and $\mathcal{N}$. See [8, 7.2.4]. In particular, since $\mathrm{VN}(G \times G) \cong \mathrm{VN}(G) \bar{\otimes} \mathrm{VN}(G)$ spatially, via the unitary which implements $\mathrm{L}^{2}(G) \otimes \mathrm{L}^{2}(G) \cong \mathrm{L}^{2}(G \times G)$, we thus have that $\mathrm{A}(G) \widehat{\otimes} \mathrm{A}(G) \cong \mathrm{A}(G \times G)$ completely isometrically. This identity holds isometrically for the Banach space projective tensor product only when $G$ is Abelian. See [20].

If $\mathcal{A}$ is an operator space which is also an algebra, it is called a completely contractive Banach algebra if the multiplication map $m_{0}: \mathcal{A} \otimes \mathcal{A} \rightarrow \mathcal{A}$ extends to a complete contraction $m: \mathcal{A} \widehat{\otimes} \mathcal{A} \rightarrow \mathcal{A}$. The Fourier algebra $\mathrm{A}(G)$ is a completely contractive Banach algebra since the multiplication map $m: \mathrm{A}(G) \widehat{\otimes} \mathrm{A}(G) \rightarrow \mathrm{A}(G)$ corresponds to restriction to the diagonal subgroup, that is, the map $R: \mathrm{A}(G \times$ $G) \rightarrow \mathrm{A}(G)$ given by $R u(s)=u(s, s)(s \in G)$. Since the adjoint $R^{*}: \operatorname{VN}(G) \rightarrow$ $\mathrm{VN}(G \times G)$ is a ${ }^{*}$-homomorphism, it is a complete contraction and hence $R \cong m$ is a complete contraction.

If $\mathcal{A}$ is a completely contractive Banach algebra and $\mathcal{X}$ is an operator space which is also an $\mathcal{A}$-module for which the module multiplication maps $m_{l, 0}: \mathcal{A} \otimes \mathcal{X} \rightarrow \mathcal{X}$ and $m_{r, 0}: \mathcal{X} \otimes \mathcal{A} \rightarrow \mathcal{X}$ extend to complete contractions $m_{l}: \mathcal{A} \widehat{\otimes} \mathcal{X} \rightarrow \mathcal{X}$ and $m_{r}: \mathcal{X} \widehat{\otimes} \mathcal{A} \rightarrow \mathcal{X}$, then $\mathcal{X}$ is called a completely contractive $\mathcal{A}$-module. A linear map $D: \mathcal{A} \rightarrow \mathcal{X}$ is called a derivation if $D(a b)=a \cdot D(b)+D(a) \cdot b$ for $a, b$ in $\mathcal{A}$. If $\mathcal{X}$ is a completely contractive $\mathcal{A}$-module, then $\mathcal{X}^{*}$ is, too. $\mathcal{A}$ is called operator amenable if every completely bounded derivation $D: \mathcal{A} \rightarrow \mathcal{X}^{*}$, where $\mathcal{X}$ is a completely contractive $\mathcal{A}$-module, is inner (i.e. $D(a)=a \cdot f-f \cdot a$ for some $f$ in $\mathcal{X}^{*}$ ). $\mathcal{A}$ is called operator weakly amenable if every completely bounded derivation $D: \mathcal{A} \rightarrow \mathcal{A}^{*}$ is inner. If $\mathcal{A}$ is commutative, this is equivalent to saying that the only completely bounded derivation $D: \mathcal{A} \rightarrow \mathcal{X}$, where $\mathcal{X}$ is any symmetric completely contractive $\mathcal{A}$-module (i.e. $a \cdot x=x \cdot a$ for $a$ in $\mathcal{A}, x$ in $\mathcal{X}$ ), is 0 . See [2] for this result in the Banach algebra case and [12] for the adaptation to the operator theoretic setting.

\section{A theorem of Groenbaek}

In this section we adapt a theorem of Groenbaek [13] to the completely contractive Banach algebra setting. Let $\mathcal{A}$ be a completely contractive Banach algebra and $\mathcal{X}$ an operator space which is a completely contractive $\mathcal{A}$-module. Let $\mathcal{A}_{1}=\mathcal{A} \oplus_{1} \mathbb{C}$ be the unitization of $\mathcal{A}$. Then $\mathcal{X}$ is a completely contractive $\mathcal{A}_{1}$ module by setting $1 \cdot x=x$ and $x \cdot 1=x$ for $x$ in $\mathcal{X}$, where $1=0 \oplus 1$ in $\mathcal{A}_{1}$. Indeed, $\mathcal{A}_{1} \widehat{\otimes} \mathcal{X} \cong(\mathcal{A} \widehat{\otimes} \mathcal{X}) \oplus_{1} \mathcal{X}$ and the left multiplication map $m_{1}: \mathcal{A}_{1} \widehat{\otimes} \mathcal{X} \rightarrow \mathcal{X}$ corresponds to the complete contraction $m \boxplus \operatorname{id}_{\mathcal{X}}:(\mathcal{A} \widehat{\otimes} \mathcal{X}) \oplus_{1} \mathcal{X} \rightarrow \mathcal{X}$ given by $m \boxplus \operatorname{id}_{\mathcal{X}}((a \otimes x) \oplus y)=a \cdot x+y$. Similarly the right multiplication map can be extended. See [7].

The projection $\pi: \mathcal{A}_{1} \rightarrow \mathcal{A}$ is a complete quotient map. Then if we let $i: \mathcal{A} \rightarrow \mathcal{A}_{1}$ be the natural injection, we get that the identity map id $\mathcal{A}_{\hat{\otimes} \mathcal{X}}$ factors as

$$
\mathcal{A} \widehat{\otimes} \mathcal{X} \stackrel{i \otimes i d}{\longrightarrow} \mathcal{A}_{1} \widehat{\otimes} \mathcal{X} \stackrel{\pi \otimes \mathrm{id}}{\longrightarrow} \mathcal{A} \widehat{\otimes} \mathcal{X} .
$$

Hence we have for $u$ in $\mathrm{M}_{n}(\mathcal{A} \widehat{\otimes} \mathcal{X})(n \times n$-matrices over $\mathcal{A} \widehat{\otimes} \mathcal{X})$ that

$$
\|u\| \leq\|\pi \otimes \mathrm{id}\|_{c b}\left\|(i \otimes \mathrm{id})_{n} u\right\| \leq\|u\|,
$$

from which it follows that $\left\|(i \otimes \mathrm{id})_{n} u\right\|=\|u\|$, so $\mathcal{A} \widehat{\otimes} \mathcal{X}$ is completely isometrically imbedded in $\mathcal{A}_{1} \widehat{\otimes} \mathcal{X}$.

Consider the sequence

$$
0 \longrightarrow \mathcal{K} \stackrel{i}{\longrightarrow} \mathcal{A}_{1} \widehat{\otimes} \mathcal{X} \stackrel{m}{\longrightarrow} \mathcal{X} \longrightarrow 0
$$


where $m$ denotes the left module multiplication map (denoted $m_{1}$ above), $\mathcal{K}=\operatorname{ker} m$ and $i: \mathcal{K} \rightarrow \mathcal{A}_{1} \widehat{\otimes} \mathcal{X}$ is the injection. Note that $\mathcal{A}_{1} \widehat{\otimes} \mathcal{X}$ is a completely contractive $\mathcal{A}$-module via $a \cdot(b \otimes x)=(a b) \otimes x$ and $(b \otimes x) \cdot a=b \otimes(x \cdot a)$. Let

$$
[\mathcal{K} ; \mathcal{A}]=\overline{\operatorname{span}}\{a \cdot u-u \cdot a: u \in \mathcal{K} \text { and } a \in \mathcal{A}\}
$$

and note that $[\mathcal{K} ; \mathcal{A}] \subset \mathcal{K}$. The following proposition is [13, Prop. 3.1]. We rework it here to ensure that it holds in our context.

Proposition 2.1. If an operator $S$ in $\mathcal{C B}\left(\mathcal{A}_{1}, \mathcal{X}^{*}\right) \cong\left(\mathcal{A}_{1} \widehat{\otimes} \mathcal{X}\right)^{*}$ is a derivation, then it annihilates $[\mathcal{K} ; \mathcal{A}]$. In particular, 0 is the only derivation in $\mathcal{C B}\left(\mathcal{A}_{1}, \mathcal{X}^{*}\right)$ if $[\mathcal{K} ; \mathcal{A}]=\mathcal{K}$.

Proof. First note that

$$
\mathcal{K}=\left\{u-1 \otimes m(u): u \in \mathcal{A}_{1} \widehat{\otimes} \mathcal{X}\right\}=\overline{\operatorname{span}}\left\{b \otimes x-1 \otimes b \cdot x: b \in \mathcal{A}_{1} \text { and } x \in \mathcal{X}\right\} .
$$

Then for any $a$ and $b$ in $\mathcal{A}_{1}$ and $x$ in $\mathcal{X}$, we have

$$
\begin{aligned}
\langle b \otimes x-1 \otimes b \cdot x, S \cdot a-a \cdot S\rangle & =\langle x, S(a b)-a \cdot S(b)\rangle-\langle b \cdot x, S(a)-a \cdot S(1)\rangle \\
& =\langle x, S(a b)-a \cdot S(b)\rangle-\langle x, S(a) \cdot b\rangle \\
& =\langle x, S(a b)-(a \cdot S(b)+S(a) \cdot b)\rangle
\end{aligned}
$$

from which it follows that $S \cdot a-a \cdot S \in \mathcal{K}^{\perp}$ for all $a$. However, $S \cdot a-a \cdot S \in \mathcal{K}^{\perp}$ for all $a$, if and only if for all $u$ in $\mathcal{K}$,

$$
0=\langle u, S \cdot a-a \cdot S\rangle=\langle a \cdot u-u \cdot a, S\rangle .
$$

Hence we obtain the first statement of the proposition.

Suppose that $S$ in $\mathcal{C B}\left(\mathcal{A}_{1}, \mathcal{X}^{*}\right)$ is a derivation. For any $u$ in $\mathcal{A}_{1} \widehat{\otimes} \mathcal{X}$, we can write $u=(u-1 \otimes m(u))+1 \otimes m(u)$, so $\mathcal{A}_{1} \widehat{\otimes} \mathcal{X}=\mathcal{K} \oplus(1 \otimes \mathcal{X})$. (Note that $u \mapsto u-1 \otimes m(u)$ is a completely bounded projection from $\mathcal{A}_{1} \widehat{\otimes} \mathcal{X}$ onto $\mathcal{K}$, so the direct sum is one of operator spaces.) Observe that $\langle 1 \otimes x, S\rangle=\langle x, S 1\rangle=0$ for any $x$ in $\mathcal{X}$, so if $S \neq 0$, then there must be $u$ in $\mathcal{K}$ such that $\langle u, S\rangle \neq 0$. This is possible only if $[\mathcal{K} ; \mathcal{A}] \neq \mathcal{K}$.

Now we will let $\mathcal{X}=\mathcal{A}$, and $m: \mathcal{A}_{1} \widehat{\otimes} \mathcal{A}_{1} \rightarrow \mathcal{A}_{1}$ be the multiplication map. Put

$$
\mathcal{K}_{1}=\operatorname{ker} m, \quad \mathcal{K}=\mathcal{K}_{1} \cap\left(\mathcal{A}_{1} \widehat{\otimes} \mathcal{A}\right) \quad \text { and } \quad \mathcal{K}_{0}=\mathcal{K}_{1} \cap(\mathcal{A} \widehat{\otimes} \mathcal{A}) .
$$

Since $\mathcal{A} \widehat{\otimes} \mathcal{A}$ imbeds into $\mathcal{A}_{1} \widehat{\otimes} \mathcal{A}$, it is easily seen that $\mathcal{K}$ is the same as in the notation above. If $\mathcal{A}$ is commutative, then $\mathcal{K}_{1}$ and hence $\mathcal{K}$ and $\mathcal{K}_{0}$ are closed ideals in $\mathcal{A}_{1} \widehat{\otimes} \mathcal{A}_{1}$.

With only trivial modifications to his proof, we get the following theorem of Groenbaek [13].

Theorem 2.2. If $\mathcal{A}$ is a commutative completely contractive Banach algebra, then the following are equivalent:

(i) $\mathcal{A}$ is operator weakly amenable.

(ii) $[\mathcal{K} ; \mathcal{A}]=\mathcal{K}$.

(iii) $\overline{\mathcal{K}_{1}^{2}}=\mathcal{K}_{1}$.

(iv) $\overline{\mathcal{K}^{2}}=\mathcal{K}$.

(v) $\overline{\mathcal{A}^{2}}=\mathcal{A}$ and $\overline{\mathcal{K}_{0}^{2}}=\overline{(\mathcal{A} \widehat{\otimes} \mathcal{A}) \cdot \mathcal{K}_{1}}$. 
Furthermore, if $\mathcal{A}$ has a bounded approximate identity, the above conditions are equivalent to

(iv) $\overline{\mathcal{K}_{0}^{2}}=\mathcal{K}_{0}$.

\section{The Fourier ALGEBRA}

We would now like to apply the above result to the Fourier algebra $\mathrm{A}(G)$ of a locally compact group $G$. Unless it is specified otherwise, let $G$ be a non-compact locally compact group.

If $\pi: G \rightarrow \mathcal{U}\left(\mathcal{H}_{\pi}\right)$ and $\sigma: G \rightarrow \mathcal{U}\left(\mathcal{H}_{\sigma}\right)$ are continuous unitary representations of $G$, let $\pi \times \sigma: G \times G \rightarrow \mathcal{U}\left(\mathcal{H}_{\pi} \otimes \mathcal{H}_{\sigma}\right)$ be the Kronecker product of $\pi$ and $\sigma$, given by $\pi \times \sigma(s, t)=\pi(s) \otimes \sigma(t)$. We note, for future reference, that $\mathrm{VN}_{\pi} \bar{\otimes} \mathrm{VN}_{\sigma}=\mathrm{VN}_{\pi \times \sigma}$. Let $\lambda: G \rightarrow \mathcal{U}\left(\mathrm{L}^{2}(G)\right)$ be the left regular representation of $G$ and $1: G \rightarrow \mathbb{T} \cong \mathcal{U}(\mathbb{C})$ be the trivial representation.

Proposition 3.1. The representations $1 \times 1,1 \times \lambda, \lambda \times 1$ and $\lambda \times \lambda$ of $G \times G$ are all disjoint (i.e. no two of these representations have subrepresentations which are unitary equivalent).

Proof. Since $G$ is non-compact, $\lambda$ has no fixed points, for a fixed point would give a constant function in $\mathrm{A}(G)$. Hence none of $1 \times \lambda, \lambda \times 1$ or $\lambda \times \lambda$ have any fixed points for all of $G \times G$, so they are all disjoint from $1 \times 1$. $1 \times \lambda$ fixes the entire subgroup $G \times\{e\}$ while neither $\lambda \times 1$ nor $\lambda \times \lambda$ have any fixed points for that subgroup, so $1 \times \lambda$ is disjoint from $\lambda \times 1$ and $\lambda \times \lambda$. Similarly, $\lambda \times 1$ and $\lambda \times \lambda$ are disjoint.

Since $\lambda$ and 1 are disjoint representations of $G$, we find that $\mathrm{VN}_{\lambda \oplus 1} \cong \mathrm{VN}_{\lambda} \oplus_{\infty}$ $\mathrm{VN}_{1}=\operatorname{VN}(G) \oplus_{\infty} \mathbb{C}$, by (1.1), and hence obtain the completely isometric identification $\mathrm{A}(G)_{1}=\mathrm{A}(G) \oplus_{1} \mathbb{C} \cong \mathrm{A}_{\lambda \oplus 1}$. The implied map is clearly an algebra isomorphism.

If $u$ and $v$ are complex functions on $G$, let $u \times v$ denote the complex valued function on $G \times G$ given by $u \times v(s, t)=u(s) v(t)$. Also, let 1 denote the constant function on $G$ (as well as the trivial representation).

Proposition 3.2. We have the following completely isometric identifications:

(i) $\mathrm{A}(G)_{1} \widehat{\otimes} \mathrm{A}(G) \cong \mathrm{A}_{(\lambda \times \lambda) \oplus(1 \times \lambda)}=\operatorname{span}\{u, 1 \times v: u \in \mathrm{A}(G \times G)$ and $v \in$ $\mathrm{A}(G)\}$.

(ii) $\mathrm{A}(G)_{1} \widehat{\otimes} \mathrm{A}(G)_{1} \cong \mathrm{A}_{(\lambda \times \lambda) \oplus(1 \times \lambda) \oplus(\lambda \times 1) \oplus(1 \times 1)}=\operatorname{span}\{u, 1 \times v, v \times 1,1 \times 1: u \in$ $\mathrm{A}(G \times G)$ and $v \in \mathrm{A}(G)\}$.

These are implemented by algebra isomorphisms.

Proof. (i) We have $\mathrm{A}(G)_{1}^{*} \cong \mathrm{VN}_{\lambda \oplus 1}$ and $\mathrm{A}(G)^{*} \cong \mathrm{VN}(G)=\mathrm{VN}_{\lambda}$. Thus, using (1.3), (1.1) and the lemma above, we obtain

$$
\begin{aligned}
\left(\mathrm{A}(G)_{1} \widehat{\otimes} \mathrm{A}(G)\right)^{*} & \cong \mathrm{VN}_{\lambda \oplus 1} \bar{\otimes} \mathrm{VN}_{\lambda}=\left(\mathrm{VN}_{\lambda} \oplus_{\infty} \mathrm{VN}_{1}\right) \bar{\otimes} \mathrm{VN}_{\lambda} \\
& =\left(\mathrm{VN}_{\lambda} \bar{\otimes} \mathrm{VN}_{\lambda}\right) \oplus_{\infty}\left(\mathrm{VN}_{1} \bar{\otimes} \mathrm{VN}_{\lambda}\right) \\
& =\mathrm{VN}_{\lambda \times \lambda} \oplus_{\infty} \mathrm{VN}_{1 \times \lambda}=\mathrm{VN}_{(\lambda \times \lambda) \oplus(1 \times \lambda)},
\end{aligned}
$$

where the last space is the dual of $\mathrm{A}_{(\lambda \times \lambda) \oplus(1 \times \lambda)}$. The latter equality in the statement (i) is a straightforward identification of $\mathrm{A}_{(\lambda \times \lambda) \oplus(1 \times \lambda)}$ in $\mathrm{B}(G \times G)$. That the identifications are implemented by algebra isomorphisms is clear.

The proof of (ii) is similar. 
Theorem 3.3. If $G$ is a locally compact group, then $\mathrm{A}(G)$ is operator weakly amenable.

Proof. If $G$ is compact, then $\mathrm{A}(G)$ is operator amenable by [8, 16.2.3] (or Theorem 4.2, infra) and hence operator weakly amenable. Hence we are left to consider non-compact $G$. Let $\pi=(1 \times 1) \oplus(1 \times \lambda) \oplus(\lambda \times 1) \oplus(\lambda \times \lambda)$ so that $\mathrm{A}_{\pi}$ is the subalgebra of $\mathrm{B}(G \times G)$ indicated in Proposition 3.2(ii) above. In the identification $\mathrm{A}(G)_{1} \widehat{\otimes} \mathrm{A}(G)_{1} \cong \mathrm{A}_{\pi}$, the multiplication map $m: \mathrm{A}(G)_{1} \widehat{\otimes} \mathrm{A}(G)_{1} \rightarrow \mathrm{A}(G)_{1}$ corresponds to the map $R: \mathrm{A}_{\pi} \rightarrow \mathrm{A}_{\lambda \oplus 1}$, which restricts functions to the diagonal subgroup $G_{D}=\{(s, s): s \in G\} \cong G$, i.e. $R u(s)=u(s, s)$ for $s$ in $G$. Letting $\mathcal{K}_{1}$ and $\mathcal{K}_{0}$ be as in (2.2), we obtain identifications

$$
\mathcal{K}_{1} \cong \operatorname{ker} R=\operatorname{span}\left\{u-1 \times R(u), u-R(u) \times 1: u \in \mathrm{A}_{\pi}\right\}
$$

and

$$
\mathcal{K}_{0} \cong \operatorname{ker} R \cap \mathrm{A}(G \times G)=\mathrm{I}\left(G_{D}\right) .
$$

Here, $\mathrm{I}\left(G_{D}\right)$ denotes the ideal in $\mathrm{A}(G \times G)$ with hull $G_{D}$ : since $G_{D}$ is a subgroup, it is a set of spectral synthesis by [14] (or see [17]) $)^{\dagger}$. From spectral synthesis we obtain that $\overline{\mathrm{I}\left(G_{D}\right)^{2}}=\mathrm{I}\left(G_{D}\right)$ so

$$
\overline{\mathcal{K}_{0}^{2}}=\mathcal{K}_{0}
$$

Since $\mathrm{A}(G \times G)$ is an ideal in $\mathrm{A}_{\pi}$, we get

$$
(\mathrm{A}(G) \widehat{\otimes} \mathrm{A}(G)) \cdot \mathcal{K}_{1} \cong \mathrm{A}(G \times G) \cdot \operatorname{ker} R \subset \mathrm{A}(G \times G) \cap \operatorname{ker} R=\mathrm{I}\left(G_{D}\right) \cong \mathcal{K}_{0} .
$$

On the other hand, again using that $G_{D}$ is a set of spectral synthesis for $\mathrm{A}(G \times G)$,

$$
\mathcal{K}_{0} \cong \mathrm{I}\left(G_{D}\right)=\overline{\mathrm{A}(G \times G) \cdot \mathrm{I}\left(G_{D}\right)} \subset \overline{\mathrm{A}(G \times G) \cdot \operatorname{ker} R} \cong \overline{(\mathrm{A}(G) \widehat{\otimes} \mathrm{A}(G)) \cdot \mathcal{K}_{1}} .
$$

We thus have, assembling (3.1) and the inclusions (3.2) and (3.3),

$$
\overline{(\mathrm{A}(G) \widehat{\otimes} \mathrm{A}(G)) \cdot \mathcal{K}_{1}}=\overline{\mathcal{K}_{0}^{2}} \text {. }
$$

Hence condition (v) of Theorem 2.2 is satisfied, since $\overline{\mathrm{A}(G)^{2}}=\mathrm{A}(G)$ by the Tauberian Theorem for $\mathrm{A}(G)$.

If $\mathcal{A}$ is a completely contractive Banach algebra and $\varphi$ is a character of $\mathcal{A}$, then $\mathbb{C}$ can be made into an $\mathcal{A}$-module via $a \cdot z=\varphi(a) z=z \cdot a$ for $a$ in $\mathcal{A}$ and $z$ in $\mathbb{C}$. If $\varphi$ is continuous, it is automatically completely bounded, and hence $\mathbb{C}$ is a completely contractive $\mathcal{A}$-module. A point derivation is a derivation $D: \mathcal{A} \rightarrow \mathbb{C}$. If $\mathcal{A}$ admits continuous non-zero point derivations, then it is not (operator) weakly amenable.

Corollary 3.4. $\mathrm{A}(G)$ has no continuous point derivations.

In contrast to the case for $\mathrm{A}(G)$, if $G$ is Abelian and non-compact, then $\mathrm{B}(G) \cong$ $\mathrm{M}(\widehat{G})$ (the measure algebra of the non-discrete Abelian group $\widehat{G}$ ) admits continuous point derivations by [5], and hence is not (operator) weakly amenable. If $G$ is a locally compact group containing a closed non-compact Abelian subgroup $H$ such that the restriction map $R_{H}: \mathrm{B}(G) \rightarrow \mathrm{B}(H)$ is surjective, then $\mathrm{B}(G)$ is not (operator) weakly amenable. Note that if $R_{H}$ is surjective, then $R_{H}^{*}: \mathrm{W}^{*}(H) \rightarrow$ $\mathrm{W}^{*}(G)$ (enveloping von Neumann algebras) is a *homomorphism, and hence a complete contraction. See [11] for further results on the weak amenability of $\mathrm{B}(G)$.

\footnotetext{
${ }^{\dagger}$ Note added in proof: The spectral synthesis result, in full generality, can be found in [24].
} 
It has been recently announced by H. G. Dales, F. Ghahramani and A. Ya. Helemskii that the measure algebra $\mathrm{M}(G)$ has continuous point derivations whenever $G$ is not discrete. Hence we deduce that $\mathrm{M}(G)$ is weakly amenable if and only if $G$ is discrete. The reasonable dual conjecture to this is: $\mathrm{B}(G)$ is operator weakly amenable if and only if $G$ is compact.

\section{Operator amenability of the Fourier algebra}

In this section we give a quick proof that $\mathrm{A}(G)$ is operator amenable when $G$ is an amenable [SIN]-group. This proof uses elements of both [8, Sec. 16] (i.e. of [23] 3.5]) and [17, 5.3].

We say that $G$ has the small invariant neighbourhood property, or that $G$ is a [SIN]-group, if there is a neighbourhood base $\mathcal{V}$ of the identity in $G$ such that $s V s^{-1}=V$ for $V$ in $\mathcal{V}$ and $s$ in $G$, i.e. each $V$ in $\mathcal{V}$ is invariant under inner automorphisms. Any compact, Abelian or discrete group is a [SIN]-group. [SIN]groups are all unimodular. See 21] for more information.

We let $\mathrm{I}\left(G_{D}\right)$ be as in the proof of Theorem 3.3 If we let $\mathrm{I}_{0}\left(G_{D}\right)$ denote the set of functions in $\mathrm{A}(G \times G)$ which are compactly supported with support disjoint from $G_{D}$, then $\overline{\mathrm{I}_{0}\left(G_{D}\right)}=\mathrm{I}\left(G_{D}\right)$, since $G_{D}$ is a set of spectral synthesis for $\mathrm{A}(G \times G)$.

Lemma 4.1. If $G$ is a [SIN]-group, then there is a bounded net $\left\{u_{V}\right\}_{V \in \mathcal{V}}$ in $\mathrm{B}(G \times G)$ such that $u_{V}(s, s)=1$ for $s$ in $G$ and $u_{V} v \rightarrow 0$ for $v$ in $\mathrm{I}\left(G_{D}\right)$.

Proof. Let $\mathcal{V}$ be a neighbourhood base of the identity in $G$ consisting of relatively compact neighbourhoods, each of which is invariant under inner automorphisms. Let $\pi: G \times G \rightarrow \mathcal{U}\left(\mathrm{L}^{2}(G)\right)$ be given by $\pi(s, t) f=\lambda(s) \rho(t) f$ for $f$ in $\mathrm{L}^{2}(G)$, where $\lambda$ and $\rho$ are the left and right regular representations of $G$, respectively. Then $\pi$ is a continuous unitary representation of $G \times G$. For $v$ in $\mathcal{V}$, let $u_{V}=\frac{1}{\mu(V)}\left\langle\pi(\cdot) \chi_{V} \mid \chi_{V}\right\rangle$, where $\chi_{V}$ is the indicator function of $V$ and $\mu$ is the Haar measure. Then for $(s, t)$ in $G \times G$,

$$
u_{V}(s, t)=\frac{1}{\mu(V)} \int_{G} \chi_{V}\left(s^{-1} r t\right) \chi_{V}(r) d \mu(r)=\frac{\mu\left(s V t^{-1} \cap V\right)}{\mu(V)} .
$$

Clearly $u_{V}(s, s)=1$ for $s$ in $G$. Hence, since each $u_{V}$ is positive definite, we have $\left\|u_{V}\right\|=u_{V}(e, e)=1$. If $v \in \mathrm{I}_{0}\left(G_{D}\right)$, then there is $V$ in $\mathcal{V}$ such that $u_{V} v=0$. Hence $u_{V} v \rightarrow 0$ for $v$ in $\mathrm{I}\left(G_{D}\right)$, where $V$ runs through decreasing elements of $\mathcal{V}$.

We remark that if $G$ is discrete, we can use the positive definite function $\chi_{G_{D}}$ in place of the net above.

Theorem 4.2. If $G$ is an amenable [SIN]-group, then $\mathrm{A}(G)$ is operator amenable.

By [8, 16.1.4] (i.e. [23]), it suffices to show that $\mathrm{A}(G)$ has an operator bounded approximate diagonal, i.e. a bounded net $\left\{w_{\beta}\right\}_{\beta \in B}$ in $\mathrm{A}(G \times G) \cong \mathrm{A}(G) \widehat{\otimes} \mathrm{A}(G)$ such that

(i) $\left\{R w_{\beta}\right\}_{\beta \in B}$ is a bounded approximate identity for $\mathrm{A}(G)$, and

(ii) $\left\|u \times 1 w_{\beta}-w_{\beta} 1 \times u\right\| \rightarrow 0$ for all $u$ in $\mathrm{A}(G)$.

Recall that $R: \mathrm{A}(G \times G) \rightarrow \mathrm{A}(G)$ is the map $R u(s)=u(s, s)$ for $s$ in $G$. Let $\left\{u_{\alpha}\right\}_{\alpha \in A}$ be a bounded approximate identity for $\mathrm{A}(G)$ (which we can obtain since $G$ is amenable; see [19]), and $\left\{u_{V}\right\}_{V \in \mathcal{V}}$ be as in the lemma above. Let $B=A \times \mathcal{V}^{A}$ be the product directed set. For each $\beta=\left(\alpha,\left(V_{\alpha^{\prime}}\right)_{\alpha^{\prime} \in A}\right)$ in $B$ let

$$
w_{\beta}=u_{V_{\alpha}} u_{\alpha} \times u_{\alpha} .
$$


Then $R w_{\beta}=u_{\alpha}^{2}$, so (i) is satisfied. To see (ii), let $v \in \mathrm{A}(G)$, so $u_{\alpha} \times u_{\alpha}(v \times 1-1 \times v) \in$ $\mathrm{I}\left(G_{D}\right)$ for each $\alpha$, and hence

$$
\begin{aligned}
\lim _{\beta}\left\|u \times 1 w_{\beta}-w_{\beta} 1 \times u\right\| & =\lim _{\beta=\left(\alpha,\left(V_{\alpha^{\prime}}\right)_{\alpha^{\prime} \in A}\right)}\left\|u_{V_{\alpha}} u_{\alpha} \times u_{\alpha}(v \times 1-1 \times v)\right\| \\
& =\lim _{\alpha} \lim _{V}\left\|u_{V} u_{\alpha} \times u_{\alpha}(v \times 1-1 \times v)\right\| \\
& =\lim _{\alpha} 0=0 .
\end{aligned}
$$

The equality of limits at $(\dagger)$ is from [18, p. 69].

\section{REFERENCES}

1. G. Arsac, Sur l'espace de Banach engendré par les coefficients d'une représentation unitaire, Pub. Dép. Math. Lyon 13 (1976), no. 2, 1-101. MR 56:3180

2. W. G. Bade, P. C. Curtis, and H. G. Dales, Amenability and weak amenability for Beurling and Lipschitz algebras, Proc. London Math. Soc. 55 (1987), no. 3, 359-377. MR 88f:46098

3. D. P. Blecher, The standard dual of an operator space, Pacific Math. J. 153 (1992), no. 1, 15-30. MR 93d:47083

4. D. P. Blecher and V. I. Paulsen, Tensor products of operator spaces, J. Funct. Anal. 99 (1991), 262-292. MR 93d:46095

5. G. Brown and W. Moran, Point derivations on $M(G)$, Bull. London Math. Soc. 8 (1976), 57-64. MR 54:5744

6. M. Despić and F. Ghahramani, Weak amenablility of group algebras of locally compact groups, Canad. Math. Bull. 37 (1994), no. 2, 165-167. MR 95c:43003

7. E. G. Effros and Z.-J. Ruan, Operator convolution algebras: an approach to quantum groups, Unpublished.

8. _ Operator spaces, London Math. Soc. Monographs, New Series, vol. 23, Oxford University Press, New York, 2000. MR 2002a:46082

9. M. Enock and J.-M. Schwartz, Kac algebras and duality of locally compact groups, Springer, Berlin, 1992. MR 94e:46001

10. P. Eymard, L'algèbre de Fourier d'un groupe localement compact, Bull. Soc. Math. France 92 (1964), 181-236. MR 37:4208

11. B. E. Forrest, Amenability and weak amenability of the Fourier algbra, Preprint.

12. B. E. Forrest and P. J. Wood, Cohomology and the operator space structure of the Fourier algebra and its second dual, Indiana Math. J. 50 (2001), 1217-1240.

13. N. Groenbaek, A characterization of weakly amenable Banach algebras, Studia Math. 94 (1989), 149-162. MR 92a:46055

14. C. S. Herz, Harmonic synthesis for subgroups, Ann. Inst. Fourier, Grenoble 23 (1973), no. 3, 91-123. MR 50:7956

15. B. E. Johnson, Cohomology in banach algebras, Memoirs of the Amer. Math. Soc., vol. 127, 1972. MR 51:11130

16. — Weak amenability of group algebras, Bull. London Math. Soc. 23 (1991), 281-284. MR 92k:43004

17. - Non-amenability of the Fourier algebra of a compact group, J. London Math. Soc. 50 (1994), no. 2, 361-374. MR 95i:43001

18. J. L. Kelley, General topology, Grad. texts in math., vol. 27, Springer, 1955. MR 16:1136c, MR 51:6681

19. H. Leptin, Sur l'algèbre de Fourier d'un groupe localement compact, C. R. Acad. Sci. Paris, Sér. A-B 266 (1968), no. 1968, 1180-1182. MR 39:362

20. V. Losert, On tensor products of the Fourier algebras, Arch. Math. 43 (1984), 370-372. MR 87c:43004

21. T. W. Palmer, Classes of nonabelian, noncompact, locally compact groups, Rocky Mountain Math. J. 8 (1978), no. 4, 683-741. MR 81j:22003

22. G. K. Pedersen, $C^{*}$-algebras and their automorphism groups, Academic Press, New York, 1979. MR 81e:46037 
23. Z.-J. Ruan, The operator amenability of $A(G)$, Amer. J. Math. 117 (1995), 1449-1474. MR 96m:43001

24. M. Takesaki and N. Tatsuuma, Duality and Subgroups, II, J. Funct. Anal. 11 (1972), 184-190. MR 52:5865

Department of Pure Mathematics, University of Waterloo, Waterloo, Ontario, CANADA N2L 3G1

E-mail address: nspronk@math.uwaterloo.ca 